\title{
Potencial de atuação do bibliotecário em atividades de inteligência organizacional: estudo de caso na Universidade Federal Fluminense
}

\author{
The librarian's potential to act on organizational intelligence \\ activities: study of case in the Federal University of Rio de Janeiro
}

Adriana Gomes PEREIRA ${ }^{1}$

Regina de Barros CIANCONI2

\section{RESUMO}

Visando comprovar que o bibliotecário está apto a atuar em atividades de inteligência organizacional, foram identificadas as competências e habilidades essenciais para atuar em Sistemas de Inteligência 0 rganizacional e as competências e habilidades do bibliotecário, com o intuito de identificar se o bibliotecário está potencialmente capacitado para atuar nesse mercado. Foi pesquisada literatura sobre os temas envolvidos, analisado um programa de curso e aplicado questionário aos professores do curso de Biblioteconomia e Documentação da Universidade Federal Fluminense, de modo a identificar que competências e habilidades procuram formar em seus alunos. De acordo com as respostas, cerca de $70 \%$ das disciplinas de Biblioteconomia atendem às competências e habilidades demandadas pelos sistemas de inteligência organizacional. Verificou-se que o bibliotecário formado pela UFF está apto a atuar em algumas das atividades relacionadas à inteligência organizacional. Foi sugerido, porém, que o bibliotecário, por meio de cursos de pós-graduação em Inteligência 0 rganizacional, amplie sua formação profissional, fazendo desse mercado especializado uma nova área de atuação.

Palavras-chave: inteligência organizacional; competências; habilidades; bibliotecário; Curso de Biblioteconomia e Documentação-Universidade Federal Fluminense.

\section{ABSTRACT}

In order to proof that the librarian is able to act in organizational intelligence activities, there were identified the essentials competences and skills a professional needs to act on 0 rganizational Intelligence and the competences

1 Bibliotecária e Documentalista, Área de Negócio Internacional, Petrobras. Av. Republica do Chile, 500, Centro, 20031-170, Rio de Janeiro, RJ, Brasil. Correspondência para/Correspondende to: A.G. PEREIRA. E-mail: <adrianagope@ predialnet.com.br>.

2 Doutora em Ciência da Informação. Professora Adjunta, Instituto de Artes e Comunicação Social-IACS, Departamento de Ciência da Informação, Universidade Federal Fluminense. Rua Lara Vilela, 126, São Domingos, 24210-590, Niterói, RJ, Brasil. E-mail: < rcianconi@globo.com>

Recebido em 4/3/2007 e aceito para publicação em 31/10/2007. 
and skills of the librarian, in order to identify if a librarian is potentially capable to acton organizational intelligence activities. It has been done a bibliographic review related to this subject, a Librarian program course of the Universidade Federal Fluminense-UFF was analyzed and also a questionnaire was applied to the professors of the course, in order to identify which skills and competences they intend to improve in their students. According to answers, around $70 \%$ of the Library course disciplines match to skills and competences needed on organizational intelligence. So, a librarian, graduated from UFF, is able to work at some of the organizational intelligence activities. However, it was suggested that the librarian, through post-graduation courses of 0 rganizational Intelligence, extends his professional background, in order that this specialized market becomes a new area of actuation.

Keywords: organizational intelligence; competences; skills; librarian; Library and Documentation Course - Universidade Federal Fluminense.

\section{INTRO DUÇÃO}

$\mathrm{Na}$ sociedade da informação molda-se um novo cenário econômico, exigindo investimento em monitoramento sistemático das informações e incentivo à geração de novos conhecimentos, de modo a possibilitar que empresas e governos se antecipem às ameaças do mercado, identificando novas oportunidades e subsidiando decisõ es estratégicas.

Tal monitoramento vem sendo denominado na literatura "inteligência organizacional", "inteligência competitiva", "inteligência empresarial", inteligência de negócios", "inteligência corporativa" , "vigilância tecnológica", com conceitos que por vezes apresentam pequenas variações de foco. 0 termo adotado neste trabalho foi inteligência organizacional (embora a expressão inteligência competitiva - IC - seja a mais comumente usada na literatura), por ser mais abrangente, incluindo "tanto a inteligência usada com fins lucrativos, como a realizada com fins de sobrevivência organizacional ou de melhoria e manutenção da qualidade de produtos e serviços em qualquer área", como afirma Cianconi (1999, p.54).

0 uso da Inteligência 0 rganizacional proporciona às organizações um aumento da competitividade no mercado, por meio de um melhor entendimento dos competidores e do ambiente competitivo em que está inserida.

0 significado atual de competitividade engloba não somente a excelência de desempenho ou eficiência técnica das empresas ou produtos; compreende, também, a capacidade de desenvolver processos sistemáticos de busca por novas oportunidades, e superação de obstáculos técnicos e organizacionais via produção e aplicação de conhecimento (Canongia et al., 2004, p.232).
0 emprego da "inteligência" tem o papel de tornar a informação estratégica capaz de apoiar a tomada de decisão, tornando necessário que as organizações invistam em Sistemas de Inteligência O rganizacional (SIO), que requerem profissionais capacitados para o desenvolvimento de suas atividades.

Choo (1998, p.215) afirma que o bibliotecário, por seu conhecimento de organizar a informação dentro de sistemas e estruturas informacionais, de modo que facilite o seu uso, é considerado um especialista da informação. Aqueles que trabalham em organizações e centros de documentações sempre estiveram à margem das funções mais importantes das organizações ou empresas, servindo apenas como staff de apoio, porém esse profissional possui habilidades que são necessárias para efetivamente adquirir, organizar e distribuir informação e "as organizações (inteligentes) não podem se dar o luxo de prescindir de sua contribuição e participação em atividades estratégicas."

Tendo em vista a importância da competitividade das organizações públicas e privadas para 0 desenvolvimento e bem-estar de um país, foi realizado estudo que serviu de base para este artigo, apresentado à Escola de Biblioteconomia da Universidade Federal Fluminense, em 2006, orientado pela Profa. Dra. Regina de Barros Cianconi, como Trabalho de Conclusão de Curso. Seu objetivo foi identificar, no processo formativo do profissional bibliotecário, capacitação para lidar com a informação estratégica, de forma que possa atuar em Sistemas de Inteligência 0 rganizacional. Escolheu-se essa atividade por ser uma área que tem como matérias primas 0 dado, a informação e o conhecimento, elementos que são também objeto de estudo da Biblioteconomia.

De modo a confirmar a hipótese de que o profissional de Biblioteconomia recebe formação 
compatível com alguns dos perfis necessários para atuação em parte do processo de Inteligência 0 rganizacional, a metodologia utilizada compreendeu: pesquisa bibliográfica sobre Inteligência 0 rganizacional e demais conceitos envolvidos, de modo a identificar seus processos e as competências e habilidades exigidas para atuar na área.

Com base na literatura foi elaborada relação contendo as competências e habilidades requeridas aos profissionais que atuam em Inteligência 0 rganizacional.

Para identificar as competências e habilidades esperadas do profissional bibliotecário, foi elaborada outra relação, com base em diversos documentos, tais como: a Resolução CNE/CES 19/2002 (Brasil, 2002) que estabelece as Diretrizes $C$ urriculares para os cursos de Educação Superior, que orientam a formulação do projeto pedagógico; o Parecer CNE / CES 492/2001 (Brasil, 2001), que define competências e habilidades, que deverão ser desenvolvidas no bibliotecário, e a Classificação Brasileira de 0 cupações (Brasil, 2002).

Como parâmetro para análise comparativa entre as duas relações de competências e habilidades, e para verificar a efetiva formação recebida pelos bibliotecários, foi realizado um estudo exploratório no Curso de Biblioteconomia e Documentação da Universidade Federal Fluminense, através da análise dos programas de curso e de questionários aplicados aos professores. Foi solicitado que apontassem, para cada disciplina lecionada, na relação de competências e habilidades desejáveis a um bibliotecário, aquelas que procuram formar nos alunos (sem que tivessem conhecimento da outra relação contendo as habilidades e competências desejáveis para atuar em sistemas de inteligência organizacional).

As respostas obtidas dos professo res fo ram então comparadas às competências e habilidades exigidas para atuar em Inteligência 0 rganizacional, segundo a literatura.

\section{A IN TELIG ÊN CIA O RG AN IZACIO N AL}

Para Valentim et al. (2003), a Inteligência 0 rganizacional é fundamental à organização sob vários aspectos, entre os quais ajudar às pessoas a desenvolverem suas atividades profissionais e auxiliar as unidades de trabalho no planejamento das ações táticas e operacionais. Também são úteis aos setores estratégicos ao definirem suas estratégias de ação, visando ao mercado, à competitividade e à globalização. Além disso, a Inteligência 0 rganizacional supre necessidades de informação, em diferentes níveis de complexidade, da organização como um todo.

Battaglia (1999, p.204) afirma que Inteligência O rganizacional é sinônimo de capacidade de antecipar as ameaças do mercado e também de novas oportunidades, por meio da informação validada para a tomada de decisão.

De acordo com a Associação Brasileira de Analistas de Inteligência Competitiva (2003), a Inteligência Competitiva é um processo informacional pró-ativo que leva à melhor tomada de decisão, seja ela estratégica ou operacional. Consiste em um processo sistemático que a descobrir as forças que regem os negócios, a reduzir o risco e conduzir o tomador de decisão a agir antecipadamente, bem como a proteger o conhecimento gerado.

Tyson (1998, p.2) considera que a Inteligência 0 rganizacional pode ser entendida como o processo de análise da informação, envolvendo o ambiente externo da organização, aplicado a processos de tomada de decisão, tanto no nível estratégico como tácito, visando à consecução dos objetivos da organização e à criação de vantagens competitivas sustentáveis.

Pozzebon, Freitas, Petrini (1997) afirmam que a essência da inteligência organizacional é o manuseio da informação sobre mercados, clientes e concorrentes e ainda as informações sobre as tendências externas, políticas e socioeconômicas, que são oriundas tanto de comunicações verbais como escritas, tais como documentos oficiais e pessoais ou entrevistas, relatos de reuniõ es e programas de TV e rádio.

Segundo Cianconi (1999, p.54), a Inteligência 0 rganizacional consiste em uma forma específica de gestão da informação, em que a ênfase é no ambiente competitivo, no conhecimento dos aspectos que podem causar impacto nos pontos fortes e fracos das organizações, no conhecimento do perfil e da atuação dos concorrentes, assim como no mapeamento do meio para identificar sinais de mudança.

A inteligência organizacional, mediante esses diversos conceitos de diferentes autores, pode ser entendida como um processo contínuo sistemático de 
coleta, tratamento e análise da informação sobre o ambiente externo, em que a informação com valor agregado é transformada em inteligência e disseminada aos usuários do Sistema em apoio a tomadas de decisão, objetivando alcançar vantagens competitivas.

Torna-se necessário, também, conceituar os termos "gestão da informação" e "gestão do conhecimento", já que essas atividades estão estreitamente relacionadas com a Inteligência 0 rganizacional. Como afirmam Valentim etal. (2003), a gestão da informação trabalha no âmbito do conhecimento explícito, a gestão do conhecimento trabalha, principalmente, no âmbito do conhecimento tácito, e a Inteligência 0 rganizacional trabalha tanto com o conhecimento tácito quanto com o explícito, tendo como maior complexidade o fato de ter que estabelecer relações e interconexões entre as duas formas de gestão.

A gestão da informação consiste nas atividades de planejar, elaborar normas e modelos, selecionar, organizar, coordenar, controlar, processar, comunicar, disseminar e avaliar informações formais e informais conhecimento explícito. Envolve o monitoramento de informações em todo o seu ciclo e a sistematização e coordenação de esforços para regular e facilitar a aquisição, o processamento, 0 armazenamento, a disseminação e o acesso à informação, visando fazer com que as informações apóiem com efetividade as operações da organização, racionalizando os fluxos.

A gestão do conhecimento consiste em ações sistemáticas para facilitar o compartilhamento de conhecimento. Essas ações estão ligadas ao processo de criação, de organização, difusão e uso do conhecimento e envolvem políticas, metodologias e tecnologias para mapear, avaliar e compartilhar tais conhecimentos. Enquanto a $\mathrm{G}$ I tem foco em informação (envolvendo sua organização, monitoramento e recuperação) e processos (seu mapeamento e gestão dos conteúdos informacionais), a GC tem foco em pessoas (envolve as comunidades, os relacionamentos, a aprendizagem organizacional), informações e processos. (Cianconi, 2003, p.231)

Valentim e G elinski $(2005$, p.2) enfatizam que, no processo de Inteligência 0 rganizacional, a gestão do conhecimento é responsável pela administração do conhecimento produzido na organização, atuando junto aos fluxos informais, e tendo como foco o capital intelectual da organização. São elementos do capital intelectual: capital humano (conhecimento dos funcionários) e capital de relacionamento (conhecimento de clientes, parceiros, fornecedores) - ambos representam o conhecimento tácito - ; capital estrutural (produtos, patentes, documentos) - informação registrada e conhecimento explícito.

Dessa maneira, pode-se afirmar que gestão da informação e gestão do conhecimento são as bases para a Inteligência $O$ rganizacional, que é uma atividade especializada, que visa auxiliar a tomada de decisão. Seu efetivo aproveitamento subsidia a Inteligência O rganizacional, permitindo segurança nos processos decisivos.

Conforme exposto na literatura da área, Inteligência 0 rganizacional é o nome tanto da atividade, como do produto que essa atividade produz e ainda o nome da área em que ela é produzida.

Diante dos conceitos anteriormente apresentados, Valentim (2002) estabelece relações entre eles:

G estão da Informação - Foco no Negócio da O rganização e trabalha essencialmente com os fluxos formais de informação.

G estão do Conhecimento - Foco no Capital Intelectual da 0 rganização e trabalha essencialmente com os fluxos informais de informação.

Inteligência Competitiva - Foco nas Estratégias da O rganização e trabalha com os dois fluxos de informação formais e informais.

A Inteligência 0 rganizacional, portanto, referese ao monito ramento e análise de dados do ambiente da concorrência, com o objetivo de gerar informações úteis para o processo decisório e para o planejamento estratégico empresarial, levando a organização a obter visão estratégica e vantagem competitiva.

\section{O rigens}

A abordagem de Inteligência 0 rganizacional, como um processo de monitoramento de informações dentro das organizações, é ainda recente, surgindo somente na década de 1980 como uma disciplina 
capaz de integrar o planejamento estratégico e as atividades de marketing e informação. A prática de Inteligência 0 rganizacional, porém, não é nova. Sua origem remete às organizações de inteligência dos governos, quando, em meados do século $X X$, nos Estados Unidos, começou a ser utilizada com fins militares e políticos. Foi utilizada na reconstrução de países europeus e também no Japão, na mesma época e com os mesmos objetivos.

Segundo Cronin e Davenport (1993, p.8), o conceito de Inteligência aplicado à empresa vem da Inteligência Social. Tanto a Inteligência Social quanto a Inteligência 0 rganizacional fazem uso inteligente da informação para atingir determinados fins, contudo a primeira aplica-se a países e nações, e a segunda aplica os mesmos princípios às organizações. Inteligência social é o processo pelo qual uma sociedade, organização ou indivíduo faz uma varredura no ambiente, interpreta o que encontra, e constrói versões de eventos que levem a vantagem competitiva.

A Associação Brasileira de Analistas de Inteligência Competitiva (2003) também afirma que a Inteligência Competitiva tem sua origem nos métodos utiliza dos pelos órgãos de inteligência governamentais, cujo principal objetivo era identificar e avaliar informações ligadas à Defesa Nacional. As ferramentas utilizadas foram adaptadas à realida de empresarial e à nova ordem mundial, sendo também incorporadas a esse processo informacional as técnicas utilizadas por três diferentes áreas: (1) Ciência da Informação, principalmente no que diz respeito às suas ferramentas de gerenciamento de informações formais; (2) Tecnologia da Informação, enfatizando as suas ferramentas de mineração de dadose (3) Administração, representada por suas áreas de estratégia, marketing e gestão.

Conclui-se que a abordagem teórica adotada nos ambientes dos negócios é baseada na atuação militar e diplomática, de onde surgiram conceitos tais como estratégia, tática e inteligência. E assim como nessas áreas, o objetivo do uso da inteligência nas organizações é vencer os inimigos (concorrentes) e/ou sobreviver.

É importante destacar, no entanto, que, apesar de a Inteligência 0 rganizacional ser originária das atividades voltadas para a obtenção ilegal de informações secretas, como na espionagem industrial, na atualidade o conceito é considerado no mundo dos negócios de forma ética, devendo as informações serem obtidas por meios legais, através de fontes públicas de informação.

\section{A Inteligência O rganizacional no Brasil}

A Inteligência 0 rganizacional chega ao Brasil a partir da década de 1990, sendo mais difundida a partir do ano 2000. A Associação Brasileira de Analistas de Inteligência Competitiva - ABRAIC (2003) - apresenta em detalhes a evolução histórica da atividade de Inteligência 0 rganizacional no Brasil, desde a década de 90 até 0 ano de 2003.

De acordo com G omes e Braga (2001, p.91) 0 motivo que levou as organizações brasileiras a investirem em Sistemas de Inteligência 0 rganizacional foi a abertura do mercado às empresas estrangeiras, em que as subsidiárias importavam das filiais seus modelos de Inteligência 0 rganizacional, estando com isso mais bem preparadas que as organizações nacionais, que precisavam enfrentar, no mesmo nível, a forte concorrência internacional.

A maioria das organizações que praticam Inteligência 0 rganizacional no Brasil é filial de multinacionais, apesar de haver também empresas totalmente brasileiras que já perceberam a importância dessa função. A atividade de Inteligência parece ter sido adotada principalmente por grandes organizações, embora seja uma atividade importante também para as pequenas e médias (uma exceção fica por conta do Sebrae, que cuida de dar consultoria a esse segmento), na medida em que as capacita para identificar o que é relevante e de uso imediato em ações que Ihes garantam a sobrevivência.

\section{SISTEMA DE IN TELIG ÊN CIA O RG AN IZACIO NAL}

Um programa sistemático de identificação, coleta, tratamento e análise de informações sobre atividades dos concorrentes, informações tecnológicas, sobre novas tendências, etc., em que são disseminadas como inteligência aos usuários, para que estes possam 
agir a partir dela, vem sendo denominado Sistema de Inteligência Competitiva - SIC (ou SIO ). Funciona como "uma antena na identificação de novas oportunidades e sinais de mudança do ambiente". (Battaglia,1999, p.8)

Dentre os objetivos de um SIO, segundo G omes e Braga (2001, p.37), estão: antecipar mudanças no ambiente dos negócios; identificar novos e potenciais competido res; antecipar as ações dos concorrentes reais; conhecer as políticas, legislações, etc. que possam afetar o negócio, bem como auxiliar a abertura de um novo negócio e aumentar a qualidade das atividades de fusão, aquisição e alianças estratégicas, por meio da obtenção de informações mais precisas sobre as empresas.

O Sistema oferece à organização informações úteis, propiciando conhecimento sobre seus concorrentes e sobre novos competidores, aumentando sua capacidade de inovar, de manter e captar novos clientes e manter posições favoráveis num ambiente competitivo, reduzindo os riscos com a tomada de decisão.

\section{Etapas do Sistema de Inteligência O rga- nizacional}

No processo de Inteligência 0 rganizacional as informações críticas advindas das demandas da organização para se manter competitiva, são obtidas a partir da estruturação de diversas atividades, conforme G omes e Braga (2001, p.47), que utilizam um modelo dividido em cinco etapas: identificação das necessidades de informação; coleta das informações; análise das informações, disseminação e avaliação. Já Battaglia (1999, p. 206) considera quatro fases principais: planejamento e coordenação; coleta, processamento e armazenamento, análise e validação, disseminação e utilização.

Entre os modelos que permitem um diagnóstico da situação para estabelecimento das estratégias competitivas estão: Fatores C ríticos de Sucesso - FSC (Critical Success Factors - CSF), que, de acordo com Rockart (1979, p. 85), são "áreas-chave nas quais as coisas devem estar indo bem para o negócio florescer", consistindo em características, condições ou variáveis que, adequadamente gerenciadas, podem ter impacto significativo sobre a posição competitiva de uma organização, ajudando a determinar as informações requeridas e a limitar a aquisição de dados desnecessários; SWO T, que vem de S - Strength (forças), W - Weakness (fraquezas), 0 - O pportunities (oportunidades), T - Threats (ameaças); Benchmarking, que consiste na avaliação de desempenho de uma organização ou processo com base em comparação com outras organiza ções ou processos.

As etapas e passos para o processo de Inteligência estão sintetizados no Q uadro 1, a lado.

Todas são de fundamental importância para que o Sistema de Inteligência 0 rganizacional cumpra o seu ciclo até chegar à inteligência.

\section{Tipos de informações utilizadas no processo de Inteligência O rganizacional}

Para desenvolver o processo de Inteligência O rganizacional, é fundamental compreender quais são as informações estratégicas e indispensáveis a esse processo e em quais fontes podem ser adquiridas.

As fontes de informação utilizadas nos SIO s são muitas, abrangendo desde publicações a contatos pessoais. São consideradas importantes fontes os contatos diretos com o setor de negócios, que envolve clientes, fornecedores, demonstrações de vendas, distribuidores, associações comerciais, consultores, varejistas, empregados e/ou ex-empregados dos concorrentes, agências de publicidade, etc. Mastambém podem ser internas, abrangendo os setores de vendas, de pesquisa mercadológica, de planejamento, de compras, de análise de produtos concorrentes. Entre as fontes de informações publicadas, estão: periódicos do setor, material promocional das empresas, relatórios anuais, relatórios de analistas financeiros, periódicos financeiros, discursos dos gerentes, periódicos de negócios, jornais nacionais e locais, diretórios, publicações governamentais. São ainda importantes como fontes os analistas financeiros, as bases de dados eletrônicas, a Internet, bancos de investimento e comerciais, anúncios, legislação, etc.

\section{Recursos humanos envolvidos no Sistema de Inteligência O rganizacional}

Para estruturar um Sistema de Inteligência 0 rganizacional, além do investimento em tecnologias da informação e seus recursos, as organizações requerem profissionais que detenham competências e habilidades para o desempenho dessa atividade. 
Q uadro 1. As etapas de um Sistema de Inteligência 0 rganizacional.

\begin{tabular}{|c|c|}
\hline ETAPAS & PASSO S \\
\hline $\begin{array}{l}\text { Planejamento e } \\
\text { coordenação }\end{array}$ & $\begin{array}{l}\text { Consiste na identificação dos usuários, no levantamento de suas reais necessidades de } \\
\text { informação, dos procedimentos mais adequados para obtê-la e dos recursos mais apropriados } \\
\text { para coletar, armazenar e disseminar a informação.. }\end{array}$ \\
\hline $\begin{array}{l}\text { Coleta, processa- } \\
\text { mento e armazena- } \\
\text { mento da informa- } \\
\text { ção }\end{array}$ & $\begin{array}{l}\text { Identificação, classificação e obtenção das fontes de consulta. As fontes de informação podem } \\
\text { ser classificadas segundo a origem (interna ou externa à organiza ção); o conteúdo (fontes } \\
\text { primárias e secundárias); a estrutura da informação (fontes formais ou textuais ou informais) e } \\
\text { o nível de confiabilidade (informações de alto risco, de confiança subjetiva e altamente } \\
\text { confiáveis). Segundo Battaglia (1999) todas as informações que estejam relacionadas com os } \\
\text { Fatores Críticos de Sucesso (FCS) devem ser coletadas e analisadas sistematicamente, } \\
\text { organizadas, indexadas e resumidas de forma a facilitar a busca em sistemas automatizados. }\end{array}$ \\
\hline $\begin{array}{l}\text { Análise e validação } \\
\text { da informação }\end{array}$ & $\begin{array}{l}\text { Visa garantir a consistência entre as informações, pois po de haver divergências entre os dados } \\
\text { coletados em fontes diferentes. N esse caso, é necessário buscar outras fontes para se chegar } \\
\text { à informação correta e mais adequada. É nesse ponto que o analista da informação realiza uma } \\
\text { a valiação completa e confiável para que a informação se transforme em Inteligência. É nessa } \\
\text { etapa que são identificados os fatores que afetam a organização, sendo muito utilizados diversos } \\
\text { modelos e métodos, como Fatores Críticos de Sucesso (FCS), SWOT, Benchmarking para a } \\
\text { definição das necessidades dos gerentes e especialistas. As autoras enfatizam que esses modelos } \\
\text { são apenas estruturas que comportam dados e informação, pois "nada substitui a capacidade } \\
\text { humana de pensar e juntar todos esses itens em uma solução completa para o problema que } \\
\text { está sendo investigado". }\end{array}$ \\
\hline $\begin{array}{l}\text { Disseminação e } \\
\text { uso da informa ção } \\
\text { estratégica }\end{array}$ & $\begin{array}{l}\text { A disseminação e uso ocorrem apósa informação ser analisada e validada. Envolve a entrega } \\
\text { do produto gerado pelo Sistema de Inteligência O rganizacional, que é a informação analisada, } \\
\text { fechando o ciclo do processo de Inteligência. É importante que seja entregue à pessoa certa, } \\
\text { em linguagem adequada, podendo ser em forma de apresentações, boletins, resumos, relatórios, } \\
\text { ferramentas, como correio eletrônico, portais corporativos, etc., para facilitar e agilizar o } \\
\text { recebimento por parte dos usuários. }\end{array}$ \\
\hline Avaliação do SIO & $\begin{array}{l}\text { Imprescindível para garantir a sua sobrevivência e aprimoramento. É preciso avaliar o } \\
\text { desempenho de cada uma das fases que compõem o Sistema, em relação às fontes, métodos, } \\
\text { produtos, etc. Além disso, é necessário avaliar junto aos usuários do Sistema os resultados } \\
\text { obtidos com o uso dos produtos gerado pelo processo e verificar se os produtos estão de } \\
\text { acordo com as reais necessidades de informação, se a linguagem e a forma de apresentação } \\
\text { estão adequadas aos usuários. }\end{array}$ \\
\hline
\end{tabular}

Valentim (2003a) afirma que é preciso observar a formação dos profissionais que farão parte da equipe de Inteligência 0 rganizacional, pois eles influenciarão na elaboração e na qualidade dos produtos e serviços resultantes do processo. A autora sugere que as organizações devem possuir uma equipe multidisciplinar, sendo considerados por ela, em termos de formação, os seguintes perfis profissionais: formação relacionada ao negócio da organização; formação relacionada a tecnologias de informação e comunicação; formação relacionada a análises estatísticas; formação relacionada a questões da informação; formação relacionada a questões do conhecimento; formação relacionada a questões de gestão/planejamento.
Compartilham essa visão G omes e Braga (2001, p.78), ao afirmarem que o Sistema de Inteligência 0 rganizacional deve possuir um leque bem amplo de profissionais que contribuam para o seu funcionamento, pois é a combinação de vários perfis profissionais que determina o sucesso do Sistema. Enfatizam que alguns profissionais necessários podem ser terceirizados, mas os perfis profissionais do gerente e do analista de informação são indispensáveis. 0 gerente é imprescindível, pois é ele que coordena as atividades, a equipe e o processo, além de ser responsável por manter a qualidade das informações geradas pelo Sistema, por criar procedimentos para a coleta e análise de dados e informações, por interagir com os agentes de decisão entregando-Ihes as informações úteis para o processo decisório e por sensibilizar a organização sobre a importância do SIO, entre outras 
responsabilidades. Já o analista de informação é de extrema importância pelo seu conhecimento, pela sua capacidade de analisar as informações coletadas e pela sua responsabilidade em gerar os produtos finais.

Assim, a Inteligência O rganizacional tem abordagem multidisciplinar, exigindo um conjunto de conhecimentos e habilidades de procedência muito variada.

\section{A conceituação de competências e habilidades}

$\mathrm{Na}$ atualidade, alterações significativas na concepção do trabalho têm produzido um repensar em termos de competências e habilidades requeridas para o desempenho profissional. 0 conceito de competência e habilidade varia de autor para autor. Mas em geral, as definições de competências enfocam a condição da aplicação adequada de conhecimentos, habilidades, juntamente com atitudes, bem como a capacidade de exercer uma atividade. E a habilidade é considerada como algo menos amplo do que a competência. Assim, a competência estaria constituída por várias habilidades e atitudes.

Perrenoud (1999, p.7) concorda que são múltiplos os significados de competência e que não há uma visão clara a esse respeito. Para ele competência é "a capacidade de agir eficazmente em um determinado tipo de situação, apoiada em conhecimentos, mas sem limitar-se a eles".

Alessandrini (2002, p.164) afirma que a noção de competência refere-se à capacidade de compreender uma determinada situação e reagir adequadamente frente a ela. E que "competência relaciona-se ao 'saber fazer algo', que envolve uma série de habilidades".

Para Mello (2004), competência é a capacidade que as pessoas têm de mobilizar conhecimentos, valores e decisões para agir de modo pertinente numa determinada situação.

Segundo definição do Documento Básico do Exame Nacional do Ensino Médio - ENEM (Brasil, 1998) -, competências são as modalidades estruturais da inteligência, isto é, as ações e operações que as pessoas utilizam para estabelecer relações com e entre objetos, situações, fenômenos e outras pessoas que desejam conhecer. $E$ as habilidades decorrem das competências adquiridas e referem-se ao plano imediato do "saber fazer". As habilidades aperfeiçoam-se e articulam-se por meio das ações e operações, o que possibilita nova reorganização das competências.

De acordo com Ruas (2000, p.5), a questão da competência se coloca num espaço de interação entre pessoas, seus saberes e capacidades, e as organizações e suas demandas. 0 autor afirma que competência não se refere apenas ao aprendizado educacional ou profissional, "nem tampouco se reduziria ao saber, ou ao saber fazer, mas seria isso sim a capacidade de mobilizar e aplicar esses conhecimentos e capacidades numa condição particular, aonde se colocam recursos e restrições próprias à situação."

Deffune e Depresbiteris $(2000$, p.59) afirmam que, em uma profissão, competência envolve vários "saberes (conhecimentos), saber fazer (prática), saber ser (atitudes) e saber agir (mobilização de tudo para fazer algo como deve ser feito)".

Fleury $(2002$, p.56) relaciona o saber teórico (conhecimento-saber) à habilidade (tarefa-saber fazer) e ao ser (atitude-saber ser).

Q uanto ao termo habilidade, também não há um consenso entre os especialistas, mas, em geral, está mais associado ao saber fazer, estando, portanto, relacionado com a forma de execução de tarefas, na aplicação de conhecimentos, de agir, de pensar.

Moretto (1999, p.51) afirma que, em geral, a habilidade é associada ao saber fazer algo específico. Dessa maneira, identificar variáveis, relacionar informações, correlacionar, a plicar, analisar, sintetizar, avaliar, manipular com destreza são exemplos de habilidades.

A habilidade favorece a aplicação da competência e da aptidão. Está associada à capacidade de aplicar e fazer uso produtivo do conhecimento adquirido utilizando-o em uma ação, visando ao atendimento de um propósito específico. (Barbalho, 2002, p.3).

0 projeto pedagógico que está em elaboração pela UFF não separa as habilidades e competências que deverão ser desenvolvidas pelo concluinte de Biblioteconomia. 0 ptou-se, então, no presente trabalho, por não separá-las também, o que entendemos não 
prejudicar o objetivo, que é comprovar que 0 bibliotecário, por sua formação, tem condições de atuar em uma equipe de inteligência organizacional.

CO MPETÊN CIAS E HABILIDADES DO BIBLIO TECÁRIO FORMADO PELA UFF E SUA CO RRESPO N DÊN CIA CO M AS EXIG IDAS PELO S SISTEMAS DE INTELIG ÊN CIA O RG AN IZACIO NAL - SIOS

Buscou-se estabelecer comparação entre as habilidades e competências exigidas para 0 desenvolvimento de atividades de inteligência organizacional, com base na literatura da área, e as habilidades e competências do bibliotecário, obtidas a partir das descrições do Parecer CN E/C ES 492/2001 (Brasil, 2001) e da Classificação Brasileira de 0 cupações (Brasil, 2002), bem como nos programas de curso e questionários a plicados aos professo res do curso Biblioteconomia e Documentação da Universidade Federal Fluminense.

Foi traçado um paralelo entre as três variáveis, verificando-se que das 32 competências/habilidades identificadas na literatura como essenciais para atuar nos $\mathrm{SIO}$ s, cerca de $70 \%$ delas são atendidas pelas competências/habilidades que as Escolas de Biblioteconomia buscam desenvolver em seus concluintes. E, a partir das habilidades e competências assinaladas pelos professores e verificadas nos programas de curso, observou-se que 0 curso de Biblioteconomia e Documentação da UFF atende às exigências no mesmo percentual.

Cabe destacar que, para identificar as competências e habilidades dos concluintes de Biblioteconomia da UFF, somente foram analisadas as disciplinas do Departamento de Ciência da Informação, ou seja, aquelas mais diretamente relacionadas ao profissional bibliotecário, embora seu currículo seja complementado com algumas disciplinas originárias de outros cursos.

Foi elaborado quadro apresentando as competências e as habilidades essenciais requeridas pelos Sistemas de Inteligência 0 rganizacional e as habilidades e competências correlatas ou correspondentes que se procuram formar no profissional bibliotecário, além das disciplinas do curso de Biblioteconomia e Documentação da UFF que, segundo os professores e programas de curso, buscam desenvolver tais habilidades e competências nos alunos. Q uando a terminologia estava diferente, porém 0 significado era o mesmo, foi feita uma correspondência entre os termos e gerada uma outra tabela, que não apresentada neste artigo.

0 quadro 2, a seguir, mostra que são citadas pelos professores 23 competências e habilidades que se pretendem formar no perfil do bibliotecário, das 32 encontradas na literatura para os SIO s (que equivalem a $71,9 \%$ destas, portanto). São apresentadas aqui somente as que mantêm alguma correspondência entre si. Aquelas requeridas pelos $\mathrm{SIO} \mathrm{s}$, mas não citadas pelos professores estão mencionadas a seguir, constituindo habilidades e competências próprias dos gerentes e analistas de informação nos $\mathrm{SIO}$ s.

O bservou-se que algumas competências e habilidades requeridas pelo SIO s não estão inseridas no núcleo de competências e habilidades do bibliotecário, tais como: conhecer métodos e ferramentas analíticas; Identificar as barreiras no fluxo de informação nas organizações; monitorar o mercado e 0 ambiente de negócio. Conhecer as principais ferramentas de Inteligência Competitiva não foram citadas pelos docentes, assim como as habilidades de Perspicácia, 0 bservação, Intuição, Persistência, Astúcia.

Pode-se concluir que, efetivamente, não fazem parte do perfil de um bibliotecário, sendo mais adequadas ao perfil do gestor do sistema de inteligência e do analista de informações.

Percebe-se, todavia, que grande parte das competências e habilidades necessárias para 0 desenvolvimento das atividades inerentes ao processo de Inteligência 0 rganizacional coincide com aquelas recomendadas para a formação do profissional bibliotecário, especialmente aquelas relacionadas ao gerenciamento da informação. Tal percepção não significa que sejam as de maior relevância para a 10 . Procurou-se mostrar, por meio do estudo de caso, que estão contempladas, no perfil do bibliotecário formado na UFF, diversas das competências e habilidades para atuar em sistemas de inteligência organizacional, atividade esta que requer a participação de diferentes perfis profissionais. 
Quadro 2. Competências e habilidades para inteligência competitiva e sua correspondência com as competências e habilidades do bibliotecário.

\begin{tabular}{|c|c|c|c|}
\hline $\begin{array}{c}\text { COMPETÊNCIAS } \\
\text { E HABILIDADES } \\
\text { PARA SISTEMAS } \\
\text { DE } \\
\text { INTELIGÊNCIA } \\
\text { O RGAN IZACIO- } \\
\text { N AL (Segundo a } \\
\text { literatura) }\end{array}$ & $\begin{array}{l}\text { COMPETENCIAS/ } \\
\text { HABILIDADES DO } \\
\text { BIBLIOTECÁRIO (Baseado } \\
\text { no Parecer CNE/CES 492/ } \\
2001 \text { e na CBO) }\end{array}$ & $\begin{array}{c}\text { COMPETENCIAS/ } \\
\text { HABILIDADES DO } \\
\text { BIBLIO TECÁRIO } \\
\text { ( Segundo Professores e } \\
\text { Programas de Curso da UFF) }\end{array}$ & DISCIPLINAS RELACIONADAS* \\
\hline 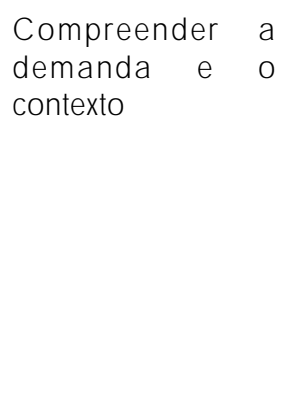 & $\begin{array}{l}\text { Traduzir as necessidades } \\
\text { dos indivíduos, grupos e } \\
\text { comunidades nas } \\
\text { respectivas áreas de } \\
\text { atuação }\end{array}$ & $\begin{array}{l}\text { Traduzir as necessidades } \\
\text { dos indivíduos, grupos e } \\
\text { comunidades nas } \\
\text { respectivas áreas de } \\
\text { atuação }\end{array}$ & $\begin{array}{l}\text { Ad.bib.II, Ad. bib.III, Bibl, Font. bibl. } \\
\text { I, Font. bibl. II, Intr. teor.inf., } \\
\text { Prod.reg. conh. II, Repr.descr.doc I, } \\
\text { Repr.descr.doc II, } \\
\text { Repr.descr.doc III, Repr.tem. I, } \\
\text { Repr.tem. II, } \\
\text { Repr.tem. III, Sel.aq.mat.doc. I, } \\
\text { Sel.aq.mat.doc. II, } \\
\text { Teor.prat. serv. ref..I, Teor.prat. serv. } \\
\text { ref.II }\end{array}$ \\
\hline $\begin{array}{l}\text { Dominar ferramen- } \\
\text { tas de acesso e } \\
\text { busca }\end{array}$ & $\begin{array}{l}\text { Elaborar estratégia de } \\
\text { busca avançada, localizar } \\
\text { e recuperar as } \\
\text { informações; processar a } \\
\text { informação registrada em } \\
\text { diferentes tipos de } \\
\text { suporte, mediante a } \\
\text { aplicação de } \\
\text { conhecimentos teóricos e } \\
\text { práticos de coleta, } \\
\text { processamento, } \\
\text { armazenamento e difusão } \\
\text { da informação. }\end{array}$ & $\begin{array}{l}\text { Elaborar estratégia de } \\
\text { busca avançada, localizar e } \\
\text { recuperar as } \\
\text { informações; processar a } \\
\text { informação registrada em } \\
\text { diferentes tipos de suporte, } \\
\text { mediante a aplicação de } \\
\text { conhecimentos teóricos e } \\
\text { práticos de coleta, } \\
\text { processamento, } \\
\text { armazenamento e difusão } \\
\text { da informação. }\end{array}$ & $\begin{array}{l}\text { Bibl, Font. bibl. I, Font. bibl. II, } \\
\text { Repr.descr.doc I, Repr.descr.doc } \\
\text { II, Repr.descr.doc III, Repr.tem. I, } \\
\text { Repr.tem. II, Repr.tem. III, } \\
\text { Sel.aq.mat.doc. I, Sel.aq.mat.doc. } \\
\text { II, Teor.prat. serv. ref..I }\end{array}$ \\
\hline $\begin{array}{l}\text { Tratar, classificar e } \\
\text { armazenar infor- } \\
\text { mações }\end{array}$ & $\begin{array}{l}\text { Catalogar, classificar, } \\
\text { indexar e armazenar } \\
\text { recursos informacionais }\end{array}$ & $\begin{array}{l}\text { Catalogar, classificar, } \\
\text { indexar e armazenar } \\
\text { recursos informacionais }\end{array}$ & $\begin{array}{l}\text { Bibl, Font. bibl. I, Font. bibl. II, } \\
\text { Repr.descr.doc I, Repr.descr.doc II, } \\
\text { Repr.descr.doc III, Repr.tem. I, } \\
\text { Repr.tem. II, Repr.tem.III }\end{array}$ \\
\hline $\begin{array}{l}\text { Selecionar e coletar } \\
\text { fontes e informações } \\
\text { relevantes }\end{array}$ & $\begin{array}{l}\text { Selecionar recursos } \\
\text { informacionais; coletar } \\
\text { informações para a } \\
\text { Instituição }\end{array}$ & $\begin{array}{l}\text { Selecionar recursos } \\
\text { informacionais; coletar } \\
\text { informações para a } \\
\text { Instituição }\end{array}$ & $\begin{array}{l}\text { Ad.bib.II, Ad. bib.III, Aut.bib, Bibl, } \\
\text { Font. bibl. I, Font. bibl. II, Intr. } \\
\text { teor.inf., Sel.aq. mat.doc. } \\
\text { I,Sel.aq.mat.doc. II, Teor.prat. serv. } \\
\text { ref..., Teor.prat. serv. ref..II }\end{array}$ \\
\hline $\begin{array}{lr}\text { Realizar análise e } \\
\text { síntese } & \text { das } \\
\text { informações } & \end{array}$ & $\begin{array}{l}\text { Demonstrar capacidade de } \\
\text { análise e síntese; elaborar } \\
\text { resenhas e resumos }\end{array}$ & $\begin{array}{l}\text { Demonstrar capacidade de } \\
\text { análise e síntese; elaborar } \\
\text { resenhas e resumos }\end{array}$ & $\begin{array}{l}\text { Ad. bib.I, Ad.bib.II, Ad. bib.III, } \\
\text { Aut.bib, Bibl, Et.prof, Intr. bib.I, Intr. } \\
\text { bib.II,Intr. teor.inf., M et.pesq.doc, } \\
\text { Prod.reg. conh. I, Prod.reg. conh. II, } \\
\text { Repr.descr.doc I,Repr.descr.doc II, } \\
\text { Repr.descr.doc III, Repr.tem. I, } \\
\text { Repr.tem. II, Repr.tem. III, } \\
\text { Sel.aq.mat.doc. I, Sel.aq.mat.doc. II, } \\
\text { Teor.prat. serv. ref..I, Teor.prat. serv. } \\
\text { ref..II }\end{array}$ \\
\hline
\end{tabular}


cont. Q uadro 2. Competências e habilidades para inteligência competitiva e sua correspondência com as competências e habilidades do bibliotecário.

\begin{tabular}{|c|c|c|c|}
\hline $\begin{array}{c}\text { CO MPETÊN CIAS } \\
\text { E HABILIDADES } \\
\text { PARA SISTEMAS } \\
\text { DE } \\
\text { INTELIG ÊN CIA } \\
\text { O RGAN IZACIO - } \\
\text { NAL (Segundo a } \\
\text { literatura) }\end{array}$ & $\begin{array}{c}\text { COMPETEN CIAS/ } \\
\text { HABILIDADES DO } \\
\text { BIBLIOTECÁRIO (Baseado } \\
\text { no Parecer CNE/CES 492/ } \\
2001 \text { e na CBO) }\end{array}$ & $\begin{array}{c}\text { CO MPETEN CIAS/ } \\
\text { HABILIDADES DO } \\
\text { BIBLIO TECÁRIO } \\
\text { ( Segundo Professores e } \\
\text { Programas de Curso da UFF) }\end{array}$ & DISCIPLINAS RELACIONADAS \\
\hline $\begin{array}{l}\text { Conhecer tecnolo- } \\
\text { gias de informação } \\
\text { e comunicação }\end{array}$ & $\begin{array}{l}\text { Desenvolver e utilizar } \\
\text { novas tecnologias; } \\
\text { avaliar e selecionar } \\
\text { tecnologias de } \\
\text { informação }\end{array}$ & $\begin{array}{l}\text { Desenvolver e utilizar } \\
\text { novas tecnologias; } \\
\text { avaliar e selecionar } \\
\text { tecnologias de } \\
\text { informação }\end{array}$ & $\begin{array}{l}\text { Aut.bib,Bibl., Cons.pres.doc., Font. } \\
\text { bibl. I, Font. bibl. II, Repr.descr.doc } \\
\text { I, Repr.descr.doc II, Repr.descr.doc } \\
\text { III, Repr.tem. I, Repr.tem. III, } \\
\text { Sel.aq.mat.doc. I, Sel.aq.mat.doc. } \\
\text { II, Teor. prat. serv. ref..I }\end{array}$ \\
\hline $\begin{array}{l}\text { Conhecer fontes/ } \\
\text { bancos/bases de } \\
\text { dados informacio- } \\
\text { nais }\end{array}$ & $\begin{array}{l}\text { Trabalhar com fontes de } \\
\text { informação de qualquer } \\
\text { natureza }\end{array}$ & $\begin{array}{l}\text { Trabalhar com fontes de } \\
\text { informação de qualquer } \\
\text { natureza }\end{array}$ & $\begin{array}{l}\text { Ad. bib.I, Ad. bib.III, Bibl, Font. } \\
\text { bibl. I, Font. bibl. II, Intr. bib.I, Intr. } \\
\text { bib.II, M et. pesq.doc, Prod.reg. } \\
\text { conh. I, Prod.reg. conh. II, } \\
\text { Repr.descr.doc I, Repr.descr.doc II, } \\
\text { Repr.descr.doc III, Repr.tem. I, } \\
\text { Repr.tem. II, Repr.tem. III, } \\
\text { Sel.aq.mat.doc. I, Sel.aq.mat.doc. } \\
\text { II, Teor.prat. serv. ref..I, Teor.prat. } \\
\text { serv. ref. .II }\end{array}$ \\
\hline $\begin{array}{l}\text { Executar buscas } \\
\text { empregando todo } \\
\text { tipo de fontes } \\
\text { (humanas, digitais, } \\
\text { físicas) }\end{array}$ & $\begin{array}{l}\text { Trabalhar com fontes de } \\
\text { informação de qualquer } \\
\text { natureza }\end{array}$ & $\begin{array}{l}\text { Trabalhar com fontes de } \\
\text { informação de qualquer } \\
\text { natureza }\end{array}$ & $\begin{array}{l}\text { Ad. bib.I, Ad. bib.III, Bibl, } \\
\text { Cons.pres.doc, Font. bibl. I, Font. } \\
\text { bibl. II, Intr. bib.I, Intr. bib.II, } \\
\text { M et.pesq.doc, Prod.reg. conh. I, } \\
\text { Prod.reg. conh. II, Repr.descr.doc I, } \\
\text { Repr.descr.doc I Repr.descr.doc II, } \\
\text { Repr.descr.doc III, Repr.tem. I, } \\
\text { Repr.tem. II, Repr.tem. III, } \\
\text { Sel.aq.mat.doc. I, Sel.aq.mat.doc. } \\
\text { II, Teor.prat. serv. ref..I, Teo r.prat. } \\
\text { serv. ref..II }\end{array}$ \\
\hline $\begin{array}{l}\text { Identificar } \\
\text { necessidades de } \\
\text { inteligência dos } \\
\text { decisores }\end{array}$ & $\begin{array}{l}\text { Elaborar estudo de perfil } \\
\text { de usuário e comunidade }\end{array}$ & $\begin{array}{l}\text { Elaborar estudo de perfil } \\
\text { de usuário e comunidade }\end{array}$ & $\begin{array}{l}\text { Ad. bib.II, Intr. teor.inf, Repr.tem. III, } \\
\text { Sel.aq.mat. doc. I, Sel.aq.mat.doc. } \\
\text { II, Teor.prat. serv. ref..I, Teo r.prat. } \\
\text { serv. ref..II }\end{array}$ \\
\hline $\begin{array}{l}\text { Planejare } \\
\text { desenvolver } \\
\text { projetos }\end{array}$ & $\begin{array}{l}\text { Elaborar, coordenar, } \\
\text { executar e avaliar planos, } \\
\text { programas e projetos; } \\
\text { formular e executar } \\
\text { políticas institucionais }\end{array}$ & $\begin{array}{l}\text { Elaborar, coordenar, } \\
\text { executar e avaliar planos, } \\
\text { programas e projetos; } \\
\text { formular e executar } \\
\text { políticas institucionais }\end{array}$ & $\begin{array}{l}\text { Ad. bib.III, Aut. bib, Bibl, } \\
\text { C ons.pres.doc., Font. bibl. II, Intr. } \\
\text { teor.inf., Repr.descr.doc III, } \\
\text { Repr.tem. II,Sel.aq.mat.doc. I, } \\
\text { Sel.aq.mat.doc. II, Teor.prat. serv. } \\
\text { ref..I, Teor.prat. serv. ref.. II }\end{array}$ \\
\hline $\begin{array}{l}\text { Agregar valor a } \\
\text { serviços e } \\
\text { produtos de } \\
\text { informação }\end{array}$ & $\begin{array}{l}\text { Interagir e agregar valor } \\
\text { nos processos de } \\
\text { geração, transferência e } \\
\text { uso da informação, em } \\
\text { qualquer ambiente. }\end{array}$ & $\begin{array}{l}\text { Interagir e agregar valor } \\
\text { nos processos de } \\
\text { geração, transferência e } \\
\text { uso da informação, em } \\
\text { qualquer ambiente. }\end{array}$ & $\begin{array}{l}\text { Ad. bib.III, Bibl Bibl, Font. bibl., } \\
\text { Font. bibl. II, Repr.descr.doc I, } \\
\text { Repr.descr.doc II, Repr.descr.doc III, } \\
\text { Repr.tem. I, Repr.tem. II, } \\
\text { Sel.aq.mat.doc. I, Sel.aq.mat.doc. } \\
\text { II, Teor. prat. serv. ref..I }\end{array}$ \\
\hline
\end{tabular}


cont. Q uadro 2. Competências e habilidades para inteligência competitiva e sua correspondência com as competências e habilidades do bibliotecário.

\begin{tabular}{|c|c|c|c|}
\hline $\begin{array}{l}\text { Disseminar } \\
\text { informação }\end{array}$ & Disseminar informação & Disseminar informação & $\begin{array}{l}\text { Ad. bib.II, Bibl, Cons.pres.doc, } \\
\text { Font. bibl. I Font. bibl. I, Font. } \\
\text { bibl. II, Intr. teor.inf., Prod.reg. } \\
\text { conh. I, Prod.reg. conh. II, } \\
\text { Repr.descr.doc I, Repr.descr.doc II, } \\
\text { Repr.descr.doc III, Repr.tem. I, } \\
\text { Repr.tem. II, Teor.prat. serv. ref..I, } \\
\text { Teor.prat. serv. ref..II }\end{array}$ \\
\hline $\begin{array}{l}\text { Analisar, } \\
\text { interpretar dados, } \\
\text { produzindo } \\
\text { informação }\end{array}$ & $\begin{array}{l}\text { G erar produtos a partir e } \\
\text { dos conhecimentos } \\
\text { adquiridos e divulgá-los. }\end{array}$ & $\begin{array}{l}\text { G erar produtos a partir de } \\
\text { dos conhecimentos } \\
\text { adquiridos e divulgá-los. }\end{array}$ & $\begin{array}{l}\text { Ad. bib.I, Ad. bib.III, Bibl, Font. } \\
\text { bibl. I, Font. bibl. II, Repr.descr.doc } \\
\text { I Repr.descr.doc I, Repr.descr.doc } \\
\text { II, Repr.descr.doc III, Repr.tem. I, } \\
\text { Repr.tem. II, Sel.aq.mat.doc. I, } \\
\text { Sel.aq.mat.doc. II, Teor.prat. serv. } \\
\text { ref..I, Teor.prat. serv. ref..II }\end{array}$ \\
\hline $\begin{array}{l}\text { Desenvolver } \\
\text { hipóteses e } \\
\text { formular perguntas }\end{array}$ & $\begin{array}{l}\text { Elaborar trabalhos técnico- } \\
\text { científicos; Ela borar } \\
\text { pesquisas temáticas. }\end{array}$ & $\begin{array}{l}\text { Elaborar trabalhos } \\
\text { técnico-científicos; } \\
\text { Elaborar pesquisas } \\
\text { temáticas }\end{array}$ & $\begin{array}{l}\text { Font. bibl. II, M et. pesq.doc, } \\
\text { Repr.descr.doc I, Repr.descr.doc II, } \\
\text { Repr.descr.doc III, Repr.tem. I, } \\
\text { Repr.tem. II, Sel.aq.mat.doc. I, } \\
\text { Sel.aq.mat.doc. II, Teor.prat. serv. } \\
\text { ref..I }\end{array}$ \\
\hline $\begin{array}{l}\text { Desenvolver } \\
\text { comunicação } \\
\text { verbal e escrita }\end{array}$ & $\begin{array}{l}\text { Demonstrar capacidade de } \\
\text { comunicação; demonstrar } \\
\text { conhecimento de outros } \\
\text { idiomas }\end{array}$ & $\begin{array}{l}\text { Demonstrar capacidade de } \\
\text { comunicação; demonstrar } \\
\text { conhecimento de outros } \\
\text { idiomas }\end{array}$ & $\begin{array}{l}\text { Ad. bib.I, Ad. bib.II, Ad. bib.III, } \\
\text { Bibl, Font. bibl. I, Font. bibl. II, } \\
\text { Intr. bib.I, Intr. bib.II,Intr. teor. inf., } \\
\text { M et. pesq. doc, Prod.reg. conh. II, } \\
\text { Repr.descr.doc I, Repr.descr.doc II, } \\
\text { Repr.descr.doc III Repr.tem. I, } \\
\text { Repr.tem. II, Repr.tem. I II, } \\
\text { Sel.aq.mat.doc. I, Sel.aq.mat.doc. } \\
\text { II, Teor.prat. serv. ref..I, Teor. prat. } \\
\text { serv. ref..II }\end{array}$ \\
\hline Criatividade & Criatividade & Criatividade & $\begin{array}{l}\text { Ad. bib.I, Ad. bib.II, Ad. bib.III, } \\
\text { Bibl, Font. bibl. I, Font. bibl. II, } \\
\text { Intr. bib.I, Intr. bib.II,Intr. teor.inf., } \\
\text { M et. pesq. doc, Prod.reg. conh. I, } \\
\text { Prod.reg. conh. II, Repr.descr.doc } \\
\text { I, Repr.descr.doc II, Repr.descr.doc } \\
\text { III, Repr.tem. I, Repr.tem. II, } \\
\text { Repr.tem. III, Sel.aq.mat.doc. I, } \\
\text { Sel.aq. mat.doc. II, Teor.prat. serv. } \\
\text { ref. .I, Teor.prat. serv. ref. .II }\end{array}$ \\
\hline Senso crítico & Senso crítico & Senso crítico & $\begin{array}{l}\text { Ad. bib.I, Ad. bib.II, Ad. bib.III, } \\
\text { Aut. bib, Cons.pres.doc., Et.prof, } \\
\text { Font. bibl. I, Font. bibl. II, Intr. } \\
\text { bib.I, Intr. bib.II11, Intr. teor.inf., } \\
\text { M et. pesq. doc, Prod.reg. conh. I, } \\
\text { Prod.reg. conh. II, Repr.descr.doc } \\
\text { I, Repr.descr.doc II, Repr.descr.doc } \\
\text { III, Repr.tem. I, Repr.tem. II, } \\
\text { Repr.tem. III, Sel.aq. mat.doc. I, } \\
\text { Sel.aq. mat.doc. II, Teor.prat. serv. } \\
\text { ref..I, Teor.prat. serv. ref. .II }\end{array}$ \\
\hline
\end{tabular}


cont. Quadro 2. Competências e habilidades para inteligência competitiva e sua correspondência com as competências e habilidades do bibliotecário.

\begin{tabular}{|c|c|c|c|}
\hline Postura ética & Postura ética & Postura ética & $\begin{array}{l}\text { Ad. bib. I, Ad. bib.II, Ad. bib.III, } \\
\text { Bibl, C ons.pres.doc., Et.prof, Font. } \\
\text { bibl. I, Font. bibl. II, Intr. bib.II, Intr. } \\
\text { teor.inf., M et. pesq.doc, Prod.reg. } \\
\text { conh. I, Prod.reg. conh. II, } \\
\text { Repr.descr.doc I, Repr.descr.doc II, } \\
\text { Repr.descr.doc III, Repr.tem. I, } \\
\text { Repr.tem. II, Repr.tem. III, } \\
\text { Sel.aq.mat.doc. I, Sel.aq.mat.doc. } \\
\text { I, Teor.prat. serv. ref..I, Teor.prat. } \\
\text { serv. ref..II }\end{array}$ \\
\hline $\begin{array}{l}\text { Capacidade } \\
\text { empreendedora }\end{array}$ & Empreendedorismo & Empreendedorismo & Ad. bib.I, Ad. bib.II, Ad. bib.III \\
\hline Auto didatismo & $\begin{array}{l}\text { Desenvolver atividades } \\
\text { profissionais autônomas, } \\
\text { de modo a orientar, } \\
\text { dirigir, assessorar, prestar } \\
\text { consultoria, realizar } \\
\text { perícias e emitir laudos } \\
\text { técnicos e pareceres }\end{array}$ & $\begin{array}{l}\text { Desenvolver atividades } \\
\text { profissionais autônomas, } \\
\text { de modo a orientar, } \\
\text { dirigir, assessorar, prestar } \\
\text { consultoria, realizar } \\
\text { perícias e emitir laudos } \\
\text { técnicos e pareceres }\end{array}$ & $\begin{array}{l}\text { Ad. bib.III, Bibl., Cons.pres.doc., } \\
\text { Repr.tem. II, Repr. tem. III }\end{array}$ \\
\hline $\begin{array}{l}\text { Espírito } \\
\text { investigativo }\end{array}$ & Espírito investigativo & Espírito investigativo & $\begin{array}{l}\text { Ad. bib.II,Aut. bib, Bibl, Font. bibl. I, } \\
\text { Font. bibl. II, Intr. bib.I, Intr. bib.II, } \\
\text { Intr. teor.inf.M et.pesq.doc, } \\
\text { Prod.reg. conh. II, Repr.descr.doc I, } \\
\text { Repr.descr.doc II, Repr.tem. I, } \\
\text { Repr.tem. II, Repr. tem. III, } \\
\text { Sel.aq.mat.doc. I, Sel.aq.mat.doc. } \\
\text { II Teor. prat. serv. ref..II }\end{array}$ \\
\hline Flexibilidade & $\begin{array}{l}\text { Responder às demandas } \\
\text { sociais de informação } \\
\text { produzidas pelas } \\
\text { transformações } \\
\text { tecnológicas que } \\
\text { caracterizam o mundo } \\
\text { contemporâneo. }\end{array}$ & $\begin{array}{l}\text { Responder às demandas } \\
\text { sociais de informação } \\
\text { produzidas pelas } \\
\text { transformações } \\
\text { tecnológicas que } \\
\text { caracterizam o mundo } \\
\text { contemporâneo. }\end{array}$ & $\begin{array}{l}\text { Ad. bib.II, Ad. bib.III, Bibl, } \\
\text { Cons. pres.doc, Font. bibl. I, Font. } \\
\text { bibl. II, Repr. descr.doc I, Repr. } \\
\text { descr.doc II,Repr. descr.doc III, } \\
\text { Repr. tem. I, Repr. tem. II, } \\
\text { Sel.aq.mat.doc. I, Sel.aq.mat.doc. } \\
\text { II, Teor. prat. serv. ref..I }\end{array}$ \\
\hline $\begin{array}{l}\text { G erenciar as } \\
\text { informações } \\
\text { estratégicas e } \\
\text { prioritárias }\end{array}$ & $\begin{array}{l}\text { Selecionar, coletar, } \\
\text { catalogar, classificar, } \\
\text { indexar, armazenar e } \\
\text { disseminar recursos } \\
\text { informacionais }\end{array}$ & $\begin{array}{l}\text { Selecionar, coletar, } \\
\text { catalogar, classificar, } \\
\text { indexar, armazenare } \\
\text { disseminar recursos } \\
\text { informacionais }\end{array}$ & $\begin{array}{l}\text { Ad. bib.II, Ad. bib.III, Aut.bib, Bibl, } \\
\text { Font. bibl. I, Font. bibl. II, Intr. } \\
\text { teor.inf., Prod.reg. conh. I, } \\
\text { Prod.reg. conh. II, Repr.descr.doc I, } \\
\text { Repr.descr.doc II, Repr.descr.doc } \\
\text { III, Repr.tem. I, Repr.tem. II, } \\
\text { Repr.tem. III, Sel.aq.mat.doc. I, } \\
\text { Sel.aq.mat.doc. II, Teor.prat. serv. } \\
\text { ref..I, Teor.prat. serv. ref..II }\end{array}$ \\
\hline
\end{tabular}

\section{* Legenda das disciplinas relacionadas:}

Ad bib I - ADM IN ISTRAÇÃO DE BIBLIO TECASI ; Ad.bib II - ADM IN ISTRAÇÃO DE BIBLIOTECAS I; Ad.bib III - ADM IN ISTRAÇÃO DE BIBLIO TECAS III;
Aut.bib - AUTO MAÇÃO EM BIBLIO TECAS;

Bibl. - BIBLIO G RAFIA;

Cons.pres.doc. - CONSERVAÇÃO E PRESERVAÇÃO DE DO CUMENTO S;

Et.prof. - ÉTICA PRO FISSIO NAL; 
Font.bibl. I - FO NTES BIBLIO G RÁFICAS I;

Font.bibl. II - FO NTES BIBLO G RÁFIC AS II;

Intr.bib.I - INTRUÇÃO À BIBLIO TECO NO MIA I;

Intr.bib.I - INTRO DUÇÃO À BIBLIOTECO NO MIA II; Intr.teor.inf. - INTRO DUÇÃO À TEO RIA DA INFO RMAÇÃO ; Met.pesq.doc - METO DO LO GIA DA .PESQ UISA DO CUMENTÁRIA;

Prod. reg. conh. I - PRO DUÇÃO DO S REG ISTRO S DO CONHECIMENTO I;

Prod. reg. conh. - II PRO DUÇÃO DO S REG ISTRO S DO CO NHEC. II;

Repr. descr.doc I - REPRESENTAÇÃO DESCRITIVA DE DO CUMENTO S I;

Repr .descr.doc II - REPRESENTAÇÃO DESC RITIVA DE DO CS॥;
Repr.descr.doc III - REPRESENTAÇÃO DESCRITIVA DE DOCS III;

Repr..tem. I - REPRESENTAÇÃO TEMÁTICA I; Repr. tem. II - REPRESENTAÇÃO TEMÁTIC A II; Repr. tem. III - REPRESENTAÇÃO TEMÁTICA III; Sel. aq. mat.doc. I - SELEÇÃO E AQ UISIÇÃO DE MATERIAIS DO CUMENTÁRIO S I;

Sel. aq.mat.doc. II - SELEÇÃO E AQ UISIÇÃO DE . MATERIAIS DO CUMENTÁRIO S ॥;

Teor. prat. serv. ref..I -TEO RIA E PRÁTICA DO SERVIÇO DE REFERÊNCIA I;

Teor. prat. serv. ref..I I- TEO RIA E PRÀTICA DO SERVIÇO DE REFERÊN CIA II;

\section{CONSIDERAÇÕ ES FINAIS}

Com a crescente busca pela competitividade, as organizações tendem a exigir, com agilidade, respostas relevantes a os seus objetivos e planejamento estratégico. E precisam investir em recursos que identifiquem necessidades e permitam monitorar 0 ambiente competitivo de forma eficiente e eficaz.

O Sistema de Inteligência 0 rganizacional visa atender essas necessidades informacionais da organização, tendo em suas etapas as atividades de planejamento e coordenação; coleta, processamento e armazenamento da informação; análise e validação da informação; disseminação e utilização da informação estratégica e avaliação do processo.

N esse contexto está inserido o bibliotecário, com seu conhecimento em administrar conteúdos informacionais. Ele pode apoiar a organização, visando a melhor atuação no mercado, maior capacidade de inovação e, conseqüentemente, maior vantagem competitiva.

A contribuição das tecnologias da informação e comunicação é muito importante em todas essas etapas do processo que transforma dadose informações dispersas em Inteligência O rganizacional. Porém, apesar da importância das tecnologias, nada substitui o elemento humano. E contar com profissionais capacitados pode levar as organizações a fazerem a diferença no mercado.

A equipe que atua com Inteligência 0 rganizacional deve ser multidisciplinar, com formações profissionais diversas, entre as quais citam-se: formação relacionada aos negócios, à gestão, às análises estatísticas, às tecnologias, às questões do conhecimento e às questões da informação. Existem competências e habilidades que são necessárias a to dos os profissionais, independentemente de sua formação, e outras que estão atreladas à formação profissional.

Deve ser enfatizado que, apesar de os conhecimentos do bibliotecário serem identificadas como muito importantes para esse mercado, a Biblioteconomia oferece uma formação geral para 0 desenvolvimento de atividades informacionais, enquanto a Inteligência O rganizacional é uma atividade especializada.

Sugere-se que as Escolas de Biblioteconomia ofereçam disciplinas optativas relacionadas a essa prática, para que os bibliotecários, dentro das organizações, sejam capazes de melhor compreender as técnicas e abordagens da Inteligência o rganizacional, de forma a agregar valor às atividades de gestão da informação e inteligência organizacional e à criação de produtos e senviços informacionais. Além disso, por meio de cursos de pós-graduação em Inteligência 0 rganizacional, o bibliotecário pode ampliar sua formação profissional. E com uma postura pró-ativa, posicionar-se com destaque, mostrando-se essencial para a missão da organização.

Foi possível comprovar, por meio do estudo de caso realizado na UFF, que o bibliotecário graduado naquela U niversidade tem potencial para assumir novos papéis, além dos tradicionais serviços em bibliotecas. Nos Sistemas de Inteligência $O$ rganizacional, entre outros, seus conhecimentos são relevantes, apresentando-se essa atividade como uma nova área de atuação, em potencial, para esse profissional, que, contudo, deve procurar a educação continuada para aperfeiçoamento. 
ALESSAN DRIN I, C.D. 0 desenvolvimento de competências e a participação pessoal na construção de um novo modelo educacional. In: ALESSAN DRIN I, C.D. Competências para ensinar no século XXI: a formação de professores e o desafio da avaliação. Porto Alegre: Artmed. 2002. p.157-176.

ASSO CIAÇÃO BRASILEIRA DE AN ALISTAS DE INTELIG ÊN CIA CO M PETITIVA. Brasília, 2003. Disponível em: <http:// www.abraic.org.br/ faqs.asp>. Acesso em: 15 out 2005.

BARBALHO , C.R.S. G estão baseada nas competências. In: SEMINÁRIO NACIO N ALDE BIBLIO TECAS UN IVERSITÁRIAS, 12., 2002, Recife. Anais eletrônicos. Recife: SN BU, 2002. Disponível em: < http://www.sibi.ufrj.br/snbu/snbu2002/oralpdf/26.a.pdfs . Acesso em: 18 de maio 2006.

BATTAG LIA, M.G.B. A inteligência competitiva modelando o sistema de informação de clientes: Finep. Ciência da Informação, v.29, n.2, p.200-214, 1999. Disponível em: <http:// www.scielo.br/pdf/ci/v28n2/28n2a12.pdf> Acesso em: 15 out. 2005.

BRASIL. Ministério do Trabalho e Emprego. Classificação brasileira de ocupações. Brasília, 2002. Disponível em: < http:// www.mtecbo.gov.br/index.htm>. Acesso em: 2 jun. 2006.

BRASIL. Ministério da Educação. Enem: documento básico. Brasília, DF: MEC/ INEP, 1998. Disponível em: < http:// www.inep.gov.br/basica/enem/publicacoes>. Acesso em: 2 jul. 2006.

BRASIL. Ministério da Educação. Conselho Nacional de Educação. Parecern. 0 492, de 03 de abril de 2001. Diário 0 ficial da União, Brasília, DF, 09 jul. 2001, Seção 1e, p. 50. Disponível em: <http:// www.mec.gov.br/Sesu/ftp/ces/ces0492.doc> . Acesso em: 4 jul. 2006.

BRASIL. M inistério da Educação. Conselho Nacional de Educação. Resolução CNE/CES n ${ }^{19}$, de 13 de março de 2002. Estabelece as diretrizes curricula res para os cursos de Biblioteconomia. Diário 0 ficial da União, Brasília, DF, 09 abr. 2002, Seção 1, p.34. Disponível em: < http://portal.mec.gov.br/cne/arquivos/pdf/ CES192002.pdf> . Acesso em: 4 jul.2006.

CAN O NG IA, C. etal. Foresight, inteligência competitiva e gestão do conhecimento: instrumentos para a gestão da inovação. G estão \& Produção, v.11, n.2, p.231-238, 2004. ISSN 0104530X. Disponível em: < http://www.scielo.br/pdf/gp/v11n2/ a09v11n2.pdf>. Acesso em: 18 maio 2006.

CHOO, C.W. Information management for the intelligent organization: the art of scanning the environment. 2.ed. Medford: Information Today, 1998.

CIANCO NI, R.B. Inteligência competitiva. In: CIAN CO NI, R.B. G estão da informação na sociedade do conhecimento. Brasília:
Serviço Nacional de Aprendizagem Industrial, Departamento Nacional, 1999. p.52-72.

CIAN CO NI, R.B. G estão do conhecimento: visão de indivíduos e organizações no Brasil. Tese (Doutorado em Ciência da Informação) - Escola de Comunicação, Universidade Federal do Rio de Janeiro, Rio de Janeiro, 2003.

CRO N IN, B.; DAVEN PO RT, E. Social intelligence. Annual Review of Information Science and Technology, v. 28, p.3-44, 1993.

DEFFUNE, D.; DEPRESBITERIS, L. Competências, habilidades e currículos de educação profissional: crônicas e reflexões. São Paulo: SENAC, 2000.

FLEURY, M.T.L. A gestão da competência e a estratégia organizacional. In: FLEURY, M.T.L. As pessoas na organização. São Paulo: $G$ ente, 2002, p.51-62.

G O MES, E.; BRAG A, F. Inteligência competitiva: como transformar informação em um negócio lucrativo. 2.ed. Rio de Janeiro: Campus, 2001.

MARCIAL, E. O papel do profissional da informação no trabalho de inteligência competitiva. Brasília. [s.n], 2003. Disponível em: < http://www. abraic.org.br/noticias_antigas_eib.asp>. Acesso em: 15 jun.2005.

MELLO , G.N. Afinal o que é competência. Escola on-line. Fundação Victor Civita, 2004. Disponível em:<http:// novaescola.abril.br/index.htm? ed/160_mar03/html/ com_palavra>. Acesso em: 15 jun.2006.

MO RETTO , V.P. Reflexões construtivistas sobre habilidades e competências. Dois Pontos: Teoria \& Prática em G estão, v.5, n.42, p.50-54, 1999

PERREN O UD, P. Construir as competências desde a escola. Porto Alegre: Artmed,1999.

PO ZZEBO N, M.; FREITAS, H.M.R.; PETRINI, M. Pela integração da inteligência competitiva nos Enterprise Information Systems (EIS). Ciência da Informação, v.26, n.3, 1997.

RO CKART, J.F. Chief executives define their own data needs. Harvard Business Review (article reprint), March, 1979.

RUAS, R. Atividade gerencial no século XXI e a formação de gestores: alguns nexos poucos explorados. ReAd Revista Eletrônica de Administração, V.6, N.3, 2000. Disponível em: < http:// read.adm. ufrgs. br/ read15/artigo/artigo2.htm> . Acesso em: 12 Jun. 2007.

TYSO N, K.W.M. The complete guide to competitive intelligence. Chicago, Kirk Tyson International, 1998. 
VALENTIM, M.L.P. C ompetências essenciais para atuar no processo de inteligência competitiva. [s.I]:[s.n], $2003 a$ FLEURY, Maria Tereza Lemos. Disponível em: < http:// www.ofaj.com.br/colunas_conteudo.php? cod $=78>$ Acesso em: 25 abr. 2006.

VALENTIM, M.L.P. Inteligência competitiva em organizações: dado, informação e conhecimento. DataG ramaZero, v. 3, n.4, ago. 2002. Disponível em: <http://www.dgz.org.br/ago02/ Art_02.htm> . Acesso em: 17 set. 2005.
VALENTIM, M.L.P.; G ELIN SKI, J.V.V. G estão do conhecimento como parte do processo de inteligência competitiva organizacional. Informação e Sociedade: Estudos, v.15, n.2, 2005. Disponível em: < http://periodicos.ufpb.br/ojs2/index. php/ies/article/ viewFile/31/29> Acesso em: 17 set. 2005.

VALENTIM, M. etal. 0 processo de inteligência competitiva em organizações. DataG ramaZero, v.4, n. 3, 2003. Disponível em: < http://www.dgz.org.br/jun03/Art_03.htm>. Acesso em: 17 set. 2005. 